\title{
A Randomized Controlled Trial of an Integrated Care Intervention to Increase Eligibility for Chronic Hepatitis C Treatment
}

\author{
Donna M. Evon, $\mathrm{PhD}^{1}$, Kelly Simpson, $\mathrm{PhD}^{1}$, Scott Kixmiller, MSW ${ }^{1}$, Joseph Galanko, \\ PhD ${ }^{1}$, Karen Dougherty, MSN, ANP ${ }^{1}$, Carol Golin, MD ${ }^{2,3,4}$, and Michael W. Fried, MD ${ }^{1}$ \\ ${ }^{1}$ Division of Gastroenterology and Hepatology, UNC School of Medicine, University of North \\ Carolina at Chapel Hill, Chapel Hill, North Carolina, USA \\ ${ }^{2}$ Division of General Medicine and Clinical Epidemiology, School of Medicine, University of North \\ Carolina at Chapel Hill, Chapel Hill, North Carolina, USA \\ ${ }^{3}$ Department of Health Behavior and Health Education, Gillings School of Global Public Health, \\ University of North Carolina at Chapel Hill, Chapel Hill, North Carolina, USA \\ ${ }^{4}$ Cecil G. Sheps Center for Health Services Research, University of North Carolina at Chapel Hill, \\ Chapel Hill, North Carolina, USA
}

\begin{abstract}
OBJECTIVES-Mental health and substance abuse (MH/SA) comorbidities are the most oftcited reasons for deferral from peginterferon (PegIFN) therapy for chronic hepatitis $\mathrm{C}$ virus $(\mathrm{HCV})$. We sought to determine whether an integrated care intervention (INT) for patients deferred from PegIFN owing to MH/SA could improve subsequent treatment eligibility rates.

METHODS-In this randomized controlled trial, $101 \mathrm{HCV}$ patients who were evaluated at two hepatology centers and deferred from antiviral therapy owing to MH/SA were enrolled. Participants were randomized to an INT $(N=50)$ or standard of care (SC; $N=51)$. The INT group received counseling and case management for up to 9 months. All participants underwent 3-, 6-, and 9-month clinical follow-up visits, where hepatologists, masked to group, re-evaluated patients for treatment eligibility. Standardized mood and alcohol use instruments were administered to all participants to aid clinicians in treatment decisions.
\end{abstract}

\footnotetext{
(C) 2011 by the American College of Gastroenterology

Correspondence: Donna M. Evon, PhD, Division of Gastroenterology and Hepatology, UNC School of Medicine, University of North Carolina at Chapel Hill, CB \# 7584, 8010 Burnett-Womack, Chapel Hill, North Carolina 27599, USA. donna_evon@med.unc.edu.

SUPPLEMENTARY MATERIAL is linked to the online version of the paper at http://www.nature.com/ajg

CONFLICT OF INTEREST

Potential competing interests: Donna M. Evon receives research grant support from Roche, and served on an advisory board for Vertex in the past 12 months. Michael W. Fried has had research grant funding and has served as ad hoc consultant for Roche. Dr Fried also serves as ad hoc consultant to Vertex, Merck, Tibotec, Pharmasset, GlaxoSmithkline, and Gilead. All other authors have nothing to disclose.

Guarantor of the article: Donna M. Evon, PhD.

Specific author contributions: Study design and implementation, grant funding, study supervision, data collection, manuscript writing, and revisions: Donna M. Evon; study coordination, patient recruitment, data collection, and manuscript editing and final approval: Kelly Simpson; study coordination, patient recruitment, data collection, and manuscript review and approval: Scott Kixmiller; data processing, statistical analyses, and manuscript review and approval: Joseph Galanko; patient referral, data collection, and review and approval of final manuscript: Karen Dougherty; study design, content expertise, and manuscript writing and final approval: Carol Golin; study design and supervision, patient referral, content expertise, and manuscript writing and final approval: Michael W. Fried.
} 
RESULTS-Of 101 participants, the mean age was 48 years and 50\% were men, 61\% Caucasian, and $77 \%$ genotype 1 . Patients were initially deferred owing to psychiatric issues (35\%), alcohol abuse (31\%), drug abuse (9\%), or more than one of these reasons (26\%). In an intent-to-treat analysis, $42 \%$ (21/50) of INT participants became eligible for therapy compared to $18 \%$ (9/51) of SC participants $(P=0.009$, relative risk $(\mathrm{RR})=2.38,95 \%$ confidence interval $(\mathrm{CI})(1.21,4.68))$. When baseline predictors significant at $P<0.10$ in univariate models were entered into multivariate models adjusted for treatment group, only baseline depression remained significant $(P$ $=0.05, \mathrm{RR}=0.98,95 \% \mathrm{CI}(0.96,1.00))$. With the exception of a model adjusted for genotype, treatment group remained significant in all models.

CONCLUSIONS-This trial suggests that INTs can increase eligibility for HCV treatment and expand treatment to the underserved population with $\mathrm{MH} / \mathrm{SA}$ comorbidities.

\section{INTRODUCTION}

Many patients diagnosed with chronic hepatitis $\mathrm{C}$ viral (HCV) infection are ineligible for peginterferon (PegIFN) and ribavirin treatment owing to one or more comorbid conditions that may interfere with its safe administration. This phenomenon occurs consistently across a variety of treatment settings (1-4). Although some patients are deferred owing to advanced liver disease or patient preference, the majority of patients are ineligible owing to mental health or substance abuse (MH/SA) issues (1,4-6). Moreover, very few patients who are initially deferred from treatment owing to $\mathrm{MH} / \mathrm{SA}$ ever proceed to antiviral treatment in the future $(1,2,6)$. Although most $\mathrm{MH} / \mathrm{SA}$ problems are manageable, a large number of these patients are left untreated for reasons that may include ongoing instability of $\mathrm{MH} / \mathrm{SA}$ conditions, lack of concurrent MH/SA care, or concerns regarding non-adherence, neuropsychiatric syndromes, and SA worsening during PegIFN treatment. Some of these concerns, however, have not withstood empirical scrutiny and are no longer viewed as justifiable reasons to withhold antiviral therapy (7-10). Studies have demonstrated that patients with concurrent MH/SA issues can safely and successfully complete PegIFN treatment when treatment is delivered in the context of a multidisciplinary team in which $\mathrm{MH} / \mathrm{SA}$ services are integrated with medical care (10-14). Despite this evidence, and the 2002 and 2009 treatment guidelines, patients with MH/SA issues continue to have difficulty accessing treatment $(1,4,15,16)$. A lack of integrated care models that provide MH/SA management may be one of the structural barriers responsible for the sluggish uptake of these guidelines $(14,17,18)$.

The barriers to treating $\mathrm{HCV}$ in patients with $\mathrm{MH} / \mathrm{SA}$ comorbidities are of concern from a public, as well as an individual, health perspective. $\mathrm{HCV}$ is highly prevalent in current drug users and in patients with mental illness, much more so than in the general population (1922). It has become more apparent that comprehensive team approaches will be needed to overcome barriers and increase successful treatment of the multitude of individuals with MH/SA who await treatment for this potentially life-threatening disease (23-28). Integrating $\mathrm{MH} / \mathrm{SA}$ into standard medical care may augment the treatment effectiveness of antiviral therapy and have a greater impact on public health outcomes.

Comprehensive team approaches are often referred to as "integrated models of care," and have become a relatively standard model for other chronic illnesses, such as HIV, diabetes mellitus, and cancer $(12,14,28,29)$. Integrated models of care are defined as any strategy or intervention "intended to improve the coordination of care and communication among caregivers, streamline protocols for movement across the care system, co-locate services, or deploy fully integrated service teams" ((28) pg. S228). Therefore, integrated care models can encompass everything from improving communication and collaboration between $\mathrm{MH} /$ SA specialists and HCV treatment providers to a fully integrated multidisciplinary team approach, in which hepatology and MH/SA specialists coexist in the same clinic $(14,25,26)$. 
A few examples of innovative integrated care models for $\mathrm{HCV}$ are described in the literature, and provide support for the favorable impact of such models on all aspects of disease management $(12,14,29-31)$. With the exception of one recent study (31), no randomized controlled trials (RCTs) have compared different models of care to standard care, and no study has explored the impact of such models on increasing access to care and improving treatment eligibility rates.

\section{Objectives and hypotheses}

The primary objective of this study was to determine whether a 9-month integrated care intervention (INT) could increase the proportion of $\mathrm{HCV}$ patients with MH/SA comorbidities who are deemed eligible for PegIFN treatment compared to patients who receive standard medical care. We hypothesized that a significantly higher proportion of patients randomized to the intervention would become eligible for PegIFN treatment at the end of a 9-month trial compared to patients who receive standard care.

A secondary objective of this study was to elucidate other baseline predictors of treatment eligibility. We hypothesized that certain baseline characteristics would contribute to predicting eligibility, over and above the impact of treatment condition.

\section{METHODS}

\section{Overview of study design}

This study was a multi-site, prospective, randomized, single-blinded, controlled intervention trial to evaluate the impact of an INT on treatment eligibility rates among patients deferred from antiviral therapy owing to MH/SA comorbidities. Participants $(n=101)$ were randomly assigned to standard of care (SC) or an INT, which included SC procedures plus an intervention. Participants were scheduled for follow-up clinic appointments at 3,6, and 9 months, at which time a hepatology clinician, masked to group assignment, re-evaluated patients' eligibility for PegIFN treatment. The INT group participated in monthly phone and in-person intervention sessions with the hepatology psychologist for up to 9 months. Sessions were individually tailored for each patient, and used case management and motivational-enhancement techniques (METs). The primary outcome of the study was the proportion of participants in each group who were deemed eligible by a hepatology clinician during the 9-month time period.

\section{Patient population and setting}

Participants were recruited from two outpatient specialty hepatology practices, one located at a large academic medical center and the other at an affiliated community medical center. Recruitment occurred between 2006 and 2009, and the study was completed in 2010. The majority of patients were recruited from a clinic dedicated to HCV study and supervised by a hepatologist with 21 years of experience (M.W.F.). Patients were also referred to the study for screening from seven other gastrointestinal clinicians, most of whom were other hepatology attendings with 12 years (range 5-15) of experience, on average, in HCV management. The study was approved by the Institutional Review Boards of both institutions. All participants provided written informed consent.

During initial clinic visits, all patients with HCV were routinely evaluated by a hepatology clinician for potential candidacy for PegIFN treatment. Patients who were deferred by the clinician from proceeding to PegIFN treatment owing to MH/SA comorbidities were referred and screened for study participation. Inclusion criteria included: new referral for $\mathrm{HCV}$; deferred from treatment owing to $\mathrm{MH}$ or SA issues; a confirmed diagnosis of $\mathrm{HCV}$; expressed interest in treatment; and medically cleared by the hepatologist to have no other 
contraindications for PegIFN treatment. Exclusion criteria included: diagnosis of psychotic disorders; living in a psychiatric residential facility; suicide attempt in the past 5 years; active intravenous drug use; cognitively impaired owing to brain disease or injury; advanced liver disease or other medical comorbidity that excluded them from PegIFN treatment, not interested in pursuing treatment; homelessness; no telephone access; and non-English speaking.

\section{Recruitment and randomization procedures}

Hepatology clinicians notified research coordinators of patients deferred from treatment and eligible for the trial. The coordinator screened and consented patients, and then randomly assigned them to INT or SC following a simple randomization procedure using a computergenerated list of random numbers. The allocation sequence was created by a researcher with no involvement in the trial, and the sequence concealed from study personnel until assignment occurred. When a coordinator was unavailable to recruit a patient who had expressed interest in the study, three phone calls were made to reach the patient. Patients who gave verbal consent to participate over the phone were then randomized and scheduled to attend a baseline 2 visit, at which time written consent was obtained.

\section{SC procedures}

Our comparison condition was an enhanced version of standard medical care in a hepatology clinic. Participants in both the SC and INT groups received the following standard procedures:

Baseline 1-During the first clinical visit, hepatology clinicians evaluated patients' eligibility for PegIFN treatment using the 2002 published recommendations on treatment eligibility (32). If judged to be a poor candidate for treatment owing to $\mathrm{MH} / \mathrm{SA}$ problems, the clinician counseled the patient on the rationale for deferral (e.g., significant depression, active alcohol abuse), and provided recommendations to achieve treatment eligibility (e.g., treatment for depression, cessation and treatment for alcohol abuse). Initial visits with the hepatologist may also have covered the following topics: significance of genotype; biopsy findings; review of PegIFN treatment regimen; response rates; psychiatric and constitutional side effects of PegIFN, with emphasis on psychiatric symptoms if psychiatric history present; avoidance of alcohol and illicit substances, which are known to advance cirrhosis; and screening for complications of liver disease. After this face-to-face discussion, the clinician then gave the patient specific written treatment recommendations, which if followed, should enhance his/her chances of becoming eligible for treatment through addressing the MH/SA concerns. These five Physician Treatment Recommendation handouts were developed a priori by members of the HCV treatment team to address minor and major MH concerns, minor and major alcohol abuse, and SA. See Supplementary Document 1 online for an example of a recommendation form addressing major alcohol consumption. In addition to receiving one or more handouts, participants received a book about hepatitis $\mathrm{C}$, and a follow-up appointment in 3 months. All participants completed four baseline self-report instruments, and appointments were scheduled to attend the baseline 2 research visit within 3 weeks.

Baseline 2-All participants were scheduled to undergo a Structured Clinical Interview for the DSM-IV (SCID) to determine psychiatric diagnosis. Participants in the INT condition were evaluated by the hepatology psychologist, and SC participants were evaluated by a research coordinator who received $30+\mathrm{h}$ of SCID training. After conducting a SCID, study personnel reviewed the Physician Treatment Recommendation handouts that each participant had received previously. To prevent therapy drift, coordinators were not allowed to discuss strategies or provide assistance in adhering to these treatment recommendations 
with the SC participants. The interventionist conducted the first intervention session after completing the SCID with each INT participant.

Follow-up clinic visits-All participants were scheduled for 3-, 6-, and 9-month clinic visits for re-evaluation of eligibility for PegIFN therapy with a hepatology clinician masked to group assignment. All participants were mailed an appointment slip and received an automated telephone reminder before the scheduled visit. Upon arrival to the clinic, participants completed three self-report instruments to assess alcohol use, depression, and anxiety. In addition, study personnel completed a Patient Outcome Form (see

Supplementary Document 2 online) at each visit by querying participants about actions taken to address the Physician Treatment Recommendations since the last visit. The purpose of this brief screening form was to provide standardized data to the clinician to facilitate consistent and well-informed decisions regarding treatment eligibility. The Patient Outcome Form was reviewed with the hepatology clinician before their clinical encounter with the patient. During the follow-up visit with the patient, the hepatology clinician inquired into the issues that previously precluded treatment, and to what extent the patient had made progress on following the treatment recommendations. After reviewing the data and re-evaluating the patient, the clinician made a decision about treatment eligibility. If deferred again, the participant continued in the trial and was re-evaluated at 6 and/or 9 months.

\section{INT condition}

The overarching aim of the intervention was to assist patients in adhering to the Physician Treatment Recommendations, which if followed, would ultimately improve their candidacy for PegIFN therapy. In addition to the standard procedures described above, the INT participants engaged in up to 9 months of sessions with the hepatology psychologist to assist them in addressing the Physician Treatment Recommendations regarding MH/SA concerns. The initial intervention session was conducted after the SCID at baseline 2, and then participants were scheduled for monthly intervention sessions delivered by telephone (at months 1, 2, 4, 5, 7, and 8) and in person (after clinic visit at months 3 and 6). In addition, INT participants received two reminder phone calls by the coordinator before each scheduled phone or clinic visit. For phone sessions, the psychologist made two attempts to contact the patient, and if unreachable, waited until the next scheduled phone call/visit to make contact.

The INT for this trial was developed taking into consideration patient characteristics of those evaluated at our clinical sites. The majority of the intervention was delivered over the telephone to reduce travel and financial burden. Whenever possible (in the majority of cases), the interventionist linked INT participants to local MH/SA programs to reduce travel burden and help patients establish contact with local resources for long-term management of $\mathrm{MH} / \mathrm{SA}$ disorders. In cases where patients were unable or unwilling to see a $\mathrm{MH} / \mathrm{SA}$ provider locally, the interventionist would provide monthly counsel to help participants follow the Physician Treatment Recommendations. When individuals were unable or unwilling to obtain $\mathrm{MH} / \mathrm{SA}$ services, they were encouraged to discuss pharmacological treatment with their internist, consider 12-step programs, or were aided in harm reduction efforts during monthly phone sessions.

The intervention sessions were based on three fields of research: (1) the Stages of Change model; (2) case management strategies; and (3) motivational enhancement techniques (METs). The interventionist was guided by the Stages of Change model, which posits that individuals move through a sequence of five stages as they contemplate, initiate, and maintain new behaviors $(33,34)$. Theoretically, deferred patients would be in various stages of readiness to address their problems, but we presumed that case management strategies 
and enhancing patient motivation would be critical for most $(33,34)$. Thus, the interventionist determined stage of readiness and employed counseling strategies that matched that particular stage (35). For patients who were motivated to address their problems, case management strategies were primarily employed, such as identifying appropriate local MH/SA services and assisting with referral and follow-up with MH/SA providers (36-38). Finally, we presumed many patients would be ambivalent about changing behaviors, thus a counseling style known as "METs" was most appropriate and employed with these patients. Broadly speaking, METs are clinical strategies designed to enhance motivation to make positive behavioral changes and engage ambivalent patients in a dialog that may eventually lead to making positive choices $(35,39)$. Although originally developed for problem drinkers, METs have since been successfully applied in many other patient populations and clinical settings (39).

\section{Masking procedures}

It was critical to mask hepatology clinicians to group assignment because they were responsible for making the final determination regarding treatment eligibility, the primary outcome of the study. The interactions between study personnel and clinicians when patients presented in clinic for re-evaluation posed the greatest threat to unblinding; thus, several precautions were established to minimize this threat. The importance of blinding procedures was reviewed frequently with hepatology clinicians and participants at each follow-up visit. Study personnel were vigilant to reviewing the Patient Outcome Form with the hepatology clinician in an objective and unbiased manner, and were not allowed to offer opinions regarding readiness for treatment. Discussions between study personnel and participants were held in private exam rooms, and all participants were asked not to divulge any information that could identify group assignment. When a clinician requested further clarification about the information provided by the patient, he/she was encouraged to address further inquiries with the patient. At no time during the study did a clinician or patient report comprising the masking requirements.

\section{Measures}

Baseline demographics-At baseline 1, participants completed a demographic characteristics form. Reasons for deferral and genotype were collected from medical chart review.

Depression, anxiety, and alcohol use-At baseline 1 and 3-, 6-, and 9-month followup visits, all participants completed depression, anxiety, and alcohol use instruments. The Beck Depression Inventory-II assessed depressive symptoms (40). The Spielberger Trait Anxiety Inventory assessed trait anxiety (41). The Alcohol Use Disorders Identification Test assessed frequency and severity of alcohol use (42). The baseline measures were collected to serve as covariates in regression models, and clinicians were not privy to these data. Data collected during follow-up visits were included on the Patient Outcome Form and used by the clinicians to make decisions regarding treatment eligibility.

Readiness for change-The University of Rhode Island Change Assessment Scale was used to assess individuals' readiness to address their MH or SA problem (33). All participants completed a University of Rhode Island Change Assessment Scale at baseline 1 and these data served as covariates in regression models. The University of Rhode Island Change Assessment Scale was completed only by INT participants at follow-up visits to guide the intervention.

Psychiatric diagnosis-At baseline 2, the SCID modules to diagnose mood, anxiety, alcohol, and substance use disorders were used to make psychiatric diagnoses (43). SCID 
data were collected to use as covariates in models, and for clinical purposes to make accurate diagnoses of deferral reasons and guide interventions with INT participants.

Primary outcome-The primary end point for the study was the proportion of participants in INT and SC who were deemed eligible for PegIFN treatment by masked hepatology clinicians at the end of a 9-month time period. Binary data (eligible vs. ineligible) were collected at 3-, 6-, and 9-month follow-up clinic visits. Clinicians had the following data available to them to inform their treatment decision: (i) self-report questionnaires assessing depression, anxiety, and alcohol abuse; (ii) Patient Outcome Form; (iii) physical exam/ clinical evaluation; (iv) laboratory data; and (v) urine toxicology screens (when available and appropriate).

Data analysis-During study design, estimating that after 9 months of intervention, 55\% of INT participants would be eligible for PegIFN therapy compared to $25 \%$ of SC participants, we had $83 \%$ power at $P=0.05$ to detect statistical significance between treatment groups. For the primary outcome analyses, an intent-to-treat analysis was conducted using data from all 101 randomized participants. The association between treatment group and the primary outcome (final eligibility at 9 months) was assessed with a two-sided Fisher's exact test. To determine if any group differences existed, baseline demographic, depression, anxiety, readiness for change, reasons for deferral, and provider were compared by group condition. Continuous and categorical variables were compared via $t$-test and Fisher's exact test, respectively. To determine if any baseline characteristics were predictive of PegIFN treatment eligibility after accounting for treatment condition, we ran logistic regression models with a dichotomous response and a log link to ascertain relative risk. First, each potential covariate was entered into a model by itself and the ones that resulted in $P<0.10$ were noted. Each of these covariates was then added singly to a model containing treatment condition. If any covariate had a $P<0.05$, it was included in a model with treatment condition, and then the process was repeated again, with the other variables, until no new covariates were significant at $P<0.05$.

\section{RESULTS}

\section{Patient characteristics}

Participant flowchart is depicted in Figure 1 and baseline characteristics are described in Table 1. A total of 136 patients were deferred from PegIFN treatment and referred for study screening. Of these, 35 patients were excluded. Patients who were randomized in person $(n$ $=81)$ vs. telephone $(n=20)$ did not differ significantly by treatment group or final treatment eligibility. The majority of patients (79\%) were evaluated by the same hepatology attending, whereas the remaining patients were evaluated by other hepatology clinicians. A Fisher's exact test determined that there was no statistically significant difference between treatment conditions by provider $(P=0.62)$. A total of 101 patients were randomized to treatment condition.

With regard to deferral reasons of the 101 study participants at baseline, $35 \%$ were deferred by clinicians for $\mathrm{MH}$ issues only, $31 \%$ were deferred for alcohol issues only, $9 \%$ were deferred for substance use issues only, and $26 \%$ were deferred for two or more reasons. Out of 101 participants, 62 attended the SCID assessment at baseline 2, and of these, $68 \%$ were diagnosed with lifetime or current unipolar, bipolar depression, or adjustment disorder; $39 \%$ with anxiety disorders; $66 \%$ with substance use disorders; and $82 \%$ with alcohol use disorders. Most patients had more than one lifetime or current MH/SA diagnosis. 


\section{Effect of INT on eligibility for PegIFN treatment}

In an intent-to-treat analysis, patients who participated in INT were more likely to become eligible for PegIFN treatment by the end of the 9-month trial compared to participants in SC ( $42 \%$ vs. $18 \%, P=0.009$ ) (Figure 2). Participants in the intervention condition were almost 2.4 times more likely to become eligible by 9 months $(95 \%$ confidence intervals $=1.2,4.7)$. Eight patients became eligible (6 INT, 2 SC) at the 3-month follow-up visit, 14 patients (9 INT, $5 \mathrm{SC}$ ) at 6-month visit, and 8 patients (6 INT, $2 \mathrm{SC}$ ) at 9-month visit.

\section{Patient characteristics associated with eligibility for PegIFN treatment in multivariate models}

The following baseline patient characteristics had a $P<0.10$ in bivariate tests with treatment eligibility: race; income level; genotype; depression; anxiety; and readiness for change. Baseline depression and anxiety were highly correlated $(r=0.90)$; therefore, only depression was considered for inclusion in multivariate modeling. When each of these five variables were added singly to a model that contained treatment condition, only baseline depression remained significant at $P<0.05$, although other variables approached significance (Table 2). When the remaining four variables were then added singly to a model, which then contained treatment condition and baseline depression, none remained significant at $P<0.05$. Finally, treatment condition remained statistically significant at $P<0.05$ when other variables were singly entered into the models, with the exception of a model that included genotype ( $P=$ 0.07 ; all data not shown).

\section{Preliminary results on antiviral treatment uptake}

Patients who were deemed eligible to proceed with antiviral therapy from an $\mathrm{MH} / \mathrm{SA}$ perspective are being followed in an observational study utilizing medical chart extraction. These data demonstrate the following: Of the 30 patients (21 INT, 9 SC) who were deemed eligible to proceed to antiviral therapy, 12/21 (57\%) of INT participants and 7/9 (78\%) of SC participants have started treatment $(P=0.42)$. Of the 19 patients to start treatment, 18 (95\%) have continued on treatment past week 4 (one patient discontinued at week 4 owing to increased depression and irritability). Out of the 11 patients $(2=\mathrm{SC}, 9=\mathrm{INT})$ who have yet to start treatment, chart extraction revealed the following reasons for treatment postponement: medical issues requiring further attention $(n=5)$; awaiting triple therapy $(n=$ 2); patient preference due to occupational or personal issue $(n=2)$; relapse to drugs/alcohol $(n=2)$; and lost to follow-up $(n=2)$. Patients may have had more than one reason for nontreatment. An overall comparison between the two groups indicated that $24 \%$ of the INT group have started treatment at this time (12/50) compared to $14 \%$ (7/51) of the SC condition $(P=0.21)$.

\section{DISCUSSION}

Despite an estimated 3.9 million individuals infected with hepatitis $C$ in the United States, only a small fraction have successfully undergone antiviral therapy (44-46). Barriers are pervasive at multiple junctures in the process from HCV screening to successful completion of antiviral treatment. First, significant barriers exist in proceeding from a positive screen for $\mathrm{HCV}$ to being referred to a clinic that provides antiviral therapy (46-48). Barriers, such as ongoing psychiatric and SA comorbidities, continue to exist after patients have gained entry into HCV treatment centers (1). Finally, the difficult treatment regimen and adverse side effects diminish treatment completion rates (49).

This study was an effort to overcome the most prevalent barriers at the second juncture in this funneling-down process. This randomized clinical trial evaluated the impact of an INT on eligibility for PegIFN therapy among HCV patients who were originally deferred from 
treatment owing to two highly prevalent barriers to treatment: MH and SA comorbidities.

We developed an intervention that was guided by the Stages of Change model, and employed case management and motivational-enhancing counseling strategies, which were delivered by the hepatology psychologist on a monthly basis by telephone and in person for up to 9 months. All participants in the study received enhanced SC, which included standardized screening instruments, written treatment recommendations addressing the comorbidity that led to deferral from treatment at baseline, a book on hepatitis $\mathrm{C}$, and follow-up clinic visits at 3-month intervals for re-evaluation of treatment eligibility by hepatologists. Patients who participated in the intervention were 2.4 times more likely to overcome barriers and be deemed eligible for PegIFN treatment compared to patients who received standard care. This study is the first investigation to demonstrate the efficacy of an integrated care approach on HCV treatment eligibility rates in the context of a clinical trial design.

In a previous retrospective analysis of treatment deferral rates at our hepatology clinic, only $16 \%$ of patients originally deferred from PegIFN treatment owing to MH/SA subsequently became eligible for therapy in the future (1). As we designed the current trial to of er enhanced SC procedures, we anticipated relatively higher eligibility rates ( $25 \%)$ for participants in the SC condition. However, this was not the case. Despite enhancement of standard procedures, the eligibility rate for standard care participants was not significantly higher than the results of the earlier study (18\% vs. 16\%). Thus, written treatment recommendations, educational materials, and frequently scheduled follow-up visits, while necessary, may be insuficient tactics for improving adherence to hepatologists' treatment recommendations. Clearly, additional interventions beyond these procedures will be needed to assist patients in making requisite behavioral changes to improve treatment eligibility.

Integrated care models are becoming increasingly common for the management and treatment of other conditions, such as HIV $(27,28,50)$. With increased recognition of the similarities between the HIV and HCV populations, as well as the medication regimens, a similar paradigm shift in disease management of $\mathrm{HCV}$ may be warranted $(25,28,51)$. The VA system, with an infrastructure that lends itself well to integrated care models, has taken the lead in spear-heading interdisciplinary management strategies to improve the care of veterans with $\mathrm{HCV}$ and comorbid MH/SA $(12,28)$. Several VA-based observational studies have demonstrated that veterans with $\mathrm{MH} / \mathrm{SA}$ comorbidities can access and successfully complete PegIFN therapy when managed within an integrated care context $(14,23,29,52,53)$. In one study, a psychiatric clinical nurse specialist was co-located in the HCV clinic to provide SA treatment and stabilize psychiatric symptoms, while preparing patients for antiviral therapy (29). Patients were screened for MH/SA disorders and referred as needed to the psychiatric clinical nurse specialist who provided case management, referrals, counseling, and psychotropic medications. Similar to our study, treatment was individually tailored to meet the needs of each patient. The results of their retrospective analysis were very congruent with our findings: $47 \%$ of patients who were followed by the psychiatric clinical nurse specialist became eligible for PegIFN therapy compared to $16 \%$ of patients who did not participate in the integrated care. Although these findings were very encouraging, they were limited by the retrospective, non-randomized, study design.

In this study, the intervention was designed to be individually tailored to address each patient's reason for deferral, motivation level, and availability of community resources. Although the absence of a manualized intervention may be perceived as a limitation, it is also a strength of the study as it allowed the interventionist to meet the unique needs of each patient. Given the heterogeneity of deferral reasons (i.e., psychiatric, alcohol, illicit drugs, dual diagnoses) and differing levels of patient motivation, it is imperative to match counseling strategies to each patient and their motivational level $(35,39)$. Another important 
factor to consider is the availability of MH/SA services in a patient's local community, which could be effected by health insurance status and rural vs. urban living.

This study employed standard operating procedures of clinical trial methodology and represents a significant strength of the study. The RCT design allowed us to empirically test for differences between the treatment conditions, something that previous investigations of integrated care models in HCV have been unable to do. The use of valid and reliable screening instruments increased the quality and consistency of hepatologists' treatment decisions. Standardized procedures (e.g., universal written treatment recommendations, masking procedures, standard intervals between clinic visits) reduced the risk of bias, which could have affected our primary outcome. These standardized procedures increase the credibility of our findings and instill greater confidence that the group differences in treatment eligibility were due to the intervention, and not other confounding variables.

Limitations of this study include the absence of treatment fidelity measures to assess the quality of the intervention and the possibility of experimenter bias influencing the clinicians' treatment eligibility decisions. The absence of audio-taping of intervention sessions precludes conducting qualitative analyses to better understand why the intervention worked. Despite tremendous effort to mask clinicians who were responsible for assessment of the primary outcome, it is possible that investigator-clinician interactions during follow-up visits could have influenced clinicians' decisions. However, we were vigilant to this issue from the start and instituted several procedures to minimize unblinding; at no time during the study did any clinician or patient report violation of masking procedures. Similarly, it is theoretically possible that the intervention simply coached patients to appear like appropriate candidates, without actually changing their underlying $\mathrm{MH} / \mathrm{SA}$ issues. We are currently following patients in a clinical observational study to collect treatment uptake and outcome data. Although this study is not complete, the preliminary data suggest that: (a) other non$\mathrm{MH} / \mathrm{SA}$ issues (e.g., medical issues, awaiting triple therapy) may contribute to further postponement of antiviral treatment, and (b) the majority of patients who start HCV treatment appear to tolerate and persist on the medical regimen. These limited data on treatment uptake and outcomes are based on very small numbers, and final conclusions should be based on a complete data set and after the new antiviral regimens are implemented.

This study can serve as a springboard for future investigations. Although this trial demonstrates the efficacy of a multi-component intervention, subsequent analyses need to elucidate individual components or active ingredients responsible for improvements in eligibility rates. Inclusion of treatment fidelity measures, such as audio-taping, would allow us to home in on active treatment ingredients. The cost-effectiveness of intervention delivery needs to be examined. While this interventionist was a PhD-level clinical psychologist colocated in the hepatology clinic, it is likely that others (e.g., nurses, social workers) could be trained to deliver similar interventions in community-based clinics, where it may be less feasible to staff a, MH/SA provider. Future studies should also determine the optimal length of INTs, and if different strategies work best for psychiatric vs. SA populations. Finally, investigations of novel intervention delivery methods, such as internet-based or texting approaches (54), should also be explored. It is essential that investigators interested in developing models to improve access to care do so within the unique infrastructure and configuration of the clinical environment so that procedures are feasible and sustainable. Although the development of models to fit the unique configurations of various treatment settings poses challenges for replication and generalizability in the short run, it behooves us to discover the most appropriate models of care befitting a variety of different $\mathrm{HCV}$ treatment clinics, in the long run. 
Given that a majority of HCV patients have yet to receive antiviral treatment, in large part due to $\mathrm{MH} / \mathrm{SA}$ comorbidities, novel health service approaches, such as the one tested in this trial, need to be developed to improve disease management. Reforming our current models of care is of tremendous public health importance now, as we look forward to the next generation of antiviral therapies. Clinicians should anticipate a re-emergence of patients with $\mathrm{MH} / \mathrm{SA}$ comorbidities who were previously deferred from treatment when the riskbenefit ratio dissuaded many providers and patients from attempting treatment. Triple therapy will alter the risk-benefit ratio and tip in favor of attempting treatment with a more diverse group of patients such as those included in this study. The development of innovative, cost-effective, and practical integrated care solutions is timely and urgently needed to address the scope of this public health problem. The INT developed for this trial may not generalize to all clinical settings, but will lay the groundwork for the exploration of other health service models that have the potential to increase access to, and successful treatment for a vast number of infected individuals who still await treatment.

\section{Acknowledgments}

Financial support: This study was partially supported by an investigator-initiated grant from Roche/Genetech, which had no role in design, conduct, analysis, or manuscript preparation. Funds were restricted to this study. Other support for this project came from grants from the National Center for Research Resources (KL2RR025746; D.M.E.); the Center for Gastrointestinal Biology and Disease (DK34987; J.G.); Clinical Translational Research Center Grant (M01 RR00046); Mentoring Grant (K24 DK066144-01; M.W.F.); Center for Aids Research (P30AI50410; C.G.); and an UNC Junior Faculty Development Award (D.M.E.).

\section{References}

1. Evon DM, Verma A, Dougherty KA, et al. High deferral rates and poorer treatment outcomes for HCV patients with psychiatric and substance use comorbidities. Dig Dis Sci. 2007; 52:3251-8. [PubMed: 17394072]

2. Falck-Ytter Y, Kale H, Mullen KD, et al. Surprisingly small effect of antiviral treatment in patients with hepatitis C. Ann Intern Med. 2002; 136:288-92. [PubMed: 11848726]

3. Muir AJ, Provenzale D. A descriptive evaluation of eligibility for therapy among veterans with chronic hepatitis C virus infection. J Clin Gastroenterol. 2002; 34:268-71. [PubMed: 11873110]

4. Bini EJ, Brau N, Currie S, et al. Prospective multicenter study of eligibility for antiviral therapy among 4,084 U.S. veterans with chronic hepatitis C virus infection. Am J Gastroenterol. 2005; 100:1772-9. [PubMed: 16086714]

5. Loftis JM, Matthews AM, Hauser P. Psychiatric and substance use disorders in individuals with hepatitis C: epidemiology and management. Drugs. 2006; 66:155-74. [PubMed: 16451091]

6. Rocca LG, Yawn BP, Wollan P, et al. Management of patients with hepatitis C in a community population: diagnosis, discussions, and decisions to treat. Ann Fam Med. 2004; 2:116-24. [PubMed: 15083850]

7. Edlin BR, Seal KH, Lorvick J, et al. Is it justifiable to withhold treatment for hepatitis C from illicitdrug users? N Engl J Med. 2001; 345:211-5. [PubMed: 11463019]

8. Castera L, Constant A, Henry C, et al. Impact on adherence and sustained virological response of psychiatric side effects during peginterferon and ribavirin therapy for chronic hepatitis C. Aliment Pharmacol Thr. 2006; 24:1223-30.

9. Schaefer M, Schmidt F, Folwaczny C, et al. Adherence and mental side effects during hepatitis C treatment with interferon alfa and ribavirin in psychiatric risk groups. Hepatology. 2003; 37:44351. [PubMed: 12540795]

10. Schaefer M, Heinz A, Backmund M. Treatment of chronic hepatitis $C$ in patients with drug dependence: time to change the rules? Addiction. 2004; 99:1167-75. [PubMed: 15317637]

11. Schaefer M, Hinzpeter A, Mohmand A, et al. Hepatitis C treatment in "difficult-to-treat" psychiatric patients with pegylated interferon-alpha and ribavirin: response and psychiatric side effects. Hepatology. 2007; 46:991-8. [PubMed: 17668880] 
12. Ho SB, Groessl E, Dollarhide A, et al. Management of chronic hepatitis C in veterans: the potential of integrated care models. Am J Gastroenterol. 2008; 103:1810-23. [PubMed: 18564122]

13. Litwin AH, Soloway I, Gourevitch MN. Integrating services for injection drug users infected with hepatitis $\mathrm{C}$ virus with methadone maintenance treatment: challenges and opportunities. Clin Infect Dis. 2005; 40(Suppl 5):S339-45. [PubMed: 15768345]

14. Sylvestre DL, Loftis JM, Hauser P, et al. Co-occurring hepatitis C, substance use, and psychiatric illness: treatment issues and developing integrated models of care. J Urban Health. 2004; 81:71934. [PubMed: 15466851]

15. Wagner GJ, Ryan GW. Hepatitis C virus treatment decision-making in the context of HIV coinfection: the role of medical, behavioral and mental health factors in assessing treatment readiness. AIDS. 2005; 19(Suppl 3):S190-8. [PubMed: 16251817]

16. Rifai MA, Moles JK, Short DD. Hepatitis C treatment eligibility and outcomes among patients with psychiatric illness. Psychiatr Serv. 2006; 57:570-2. [PubMed: 16603757]

17. Edlin BR, Kresina TF, Raymond DB, et al. Overcoming barriers to prevention, care, and treatment of hepatitis C in illicit drug users. Clin Infect Dis. 2005; 40(Suppl 5):S276-85. [PubMed: 15768335]

18. McLaren M, Garber G, Cooper C. Barriers to hepatitis C virus treatment in a Canadian HIVhepatitis C virus coinfection tertiary care clinic. Can J Gastroenterol. 2008; 22:133-7. [PubMed: 18299730]

19. Rosenberg SD, Goodman LA, Osher FC, et al. Prevalence of HIV, hepatitis B, and hepatitis C in people with severe mental illness. Am J Public Health. 2001; 91:31-7. [PubMed: 11189820]

20. Klinkenberg WD, Caslyn RJ, Morse GA, et al. Prevalence of human immunodeficiency virus, hepatitis $\mathrm{B}$, and hepatitis $\mathrm{C}$ among homeless persons with co-occurring severe mental illness and substance use disorders. Compr Psychiatry. 2003; 44:293-302. [PubMed: 12923707]

21. Zdilar D, Franco-Bronson K, Buchler N, et al. Hepatitis C, interferon alfa, and depression. Hepatology. 2000; 31:1207-11. [PubMed: 10827143]

22. McCarthy JJ, Flynn N. Hepatitis C in methadone maintenance patients: prevalence and public policy implications. J Addict Dis. 2001; 20:19-31. [PubMed: 11286428]

23. Fireman M, Indest DW, Blackwell A, et al. Addressing tri-morbidity (hepatitis C, psychiatric disorders, and substance use): the importance of routine mental health screening as a component of a comanagement model of care. Clin Infect Dis. 2005; 40(Suppl 5):S286-91. [PubMed: 15768336]

24. Birkhead GS, Klein SJ, Candelas AR, et al. Integrating multiple programme and policy approaches to hepatitis $\mathrm{C}$ prevention and care for injection drug users: a comprehensive approach. Int J Drug Policy. 2007; 18:417-25. [PubMed: 17854731]

25. Goldberg RW. Supported medical care: a multi-faceted approach to helping HIV/hepatitis C virus co-infected adults with serious mental illness. AIDS. 2005; 19(Suppl 3):S215-20. [PubMed: 16251821]

26. Grebely J, Knight E, Genoway KA, et al. Optimizing assessment and treatment for hepatitis C virus infection in illicit drug users: a novel model incorporating multidisciplinary care and peer support. Eur J Gastroenterol Hepatol. 2010; 22:270-7. [PubMed: 20425880]

27. Zaller N, Gillani FS, Rich JD. A model of integrated primary care for HIV-positive patients with underlying substance use and mental illness. AIDS Care. 2007; 19:1128-33. [PubMed: 18058396]

28. Willenbring ML. Integrating care for patients with infectious, psychiatric, and substance use disorders: concepts and approaches. AIDS. 2005; 19(Suppl 3):S227-37. [PubMed: 16251823]

29. Knott A, Dieperink E, Willenbring ML, et al. Integrated psychiatric/medical care in a chronic hepatitis C clinic: effect on antiviral treatment evaluation and outcomes. Am J Gastroenterol. 2006; 101:2254-62. [PubMed: 17032190]

30. Grebely J, Genoway K, Khara M, et al. Treatment uptake and outcomes among current and former injection drug users receiving directly observed therapy within a multidisciplinary group model for the treatment of hepatitis C virus infection. Int J Drug Policy. 2007; 18:437-43. [PubMed: 17854734]

31. Neri S, Bertino G, Petralia A, et al. A multidisciplinary therapeutic approach for reducing the risk of psychiatric side effects in patients with chronic hepatitis $\mathrm{C}$ treated with pegylated interferon alpha and ribavirin. J Clin Gastroenterol. 2010; 44:e210-7. [PubMed: 20838237] 
32. Seeff LB, Hoofnagle JH. National institutes of health consensus development conference: management of hepatitis C: 2002. Hepatology. 2002; 36(5, Suppl 1):S1-2. [PubMed: 12407571]

33. McConnaughy E, Prochaska J, Velicer W. Stages of change in psychotherapy: measurement and sample profiles. Psychotherapy: Theory, Research and Practice. 1983; 20:368-75.

34. Prochaska, JO.; DiClemente, CC. The Transtheoretical Approach: Crossing Traditional Boundaries of Therapy. Dow Jones-Erwin; Homewood, IL: 1984.

35. Miller, WR. Enhancing Motivation for Change in Substance Abuse Treatment. Department of Health and Human Services, Substance Abuse and Mental Health Services Administration, Center for Substance Abuse Treatment (DHHD Publication No. (SMA) 99-3354); Rockville, MD: 1999. Treatment Improvement Protocol (TIP) Series 35

36. Drake RE, O' Neal EL, Wallach MA. A systematic review of psychosocial research on psychosocial interventions for people with co-occurring severe mental and substance use disorders. J Subst Abuse Treat. 2008; 34:123-38. [PubMed: 17574803]

37. Craw JA, Gardner LI, Marks G, et al. Brief strengths-based case management promotes entry into HIV medical care: results of the antiretroviral treatment access study-II. J Acquir Immune Defic Syndr. 2008; 47:597-606. [PubMed: 18285714]

38. Husbands W, Browne G, Caswell J, et al. Case management community care for people living with HIV/AIDS (PLHAs). AIDS Care. 2007; 19:1065-72. [PubMed: 17852006]

39. Miller, WR.; Rollnick, S. Motivational Interviewing: Preparing People for Change. 2. Guilford Press; New York: 2002.

40. Beck, A.; Steer, R.; Brown, GK. Manual for Beck Depression Inventory-II. Psychological Corporation; San Antonio, TX: 1996.

41. Spielberger, C.; Gorsuch, R.; Lushene, R., et al. Manual for the State-Trait Anxiety Inventory. Mind Garden; Palo Alto, CA: 1983.

42. Saunders JB, Aasland OG, Babor TF, et al. Development of the alcohol use disorders identification test (AUDIT): WHO collaborative project on early detection of persons with harmful alcohol consumption-II. Addiction. 1993; 88:791-804. [PubMed: 8329970]

43. First, M.; Spitzer, R.; Gibbon, M., et al. Structured Clinical Interview for DSM-IV-TR Axis I Disorders, Research Version, Non-patient Edition (SCID-I/NP). Biometrics Research, New York State Psychiatric Institute; New York: 2002.

44. Alter MJ, Kruszon-Moran D, Nainan OV, et al. The prevalence of hepatitis C virus infection in the United States, 1988 through 1994. N Engl J Med. 1999; 341:556-62. [PubMed: 10451460]

45. Volk ML. Antiviral therapy for hepatitis C: why are so few patients being treated? J Antimicrob Chemother. 2010; 65:1327-9. [PubMed: 20460398]

46. Volk ML, Tocco R, Saini S, et al. Public health impact of antiviral therapy for hepatitis C in the United States. Hepatology. 2009; 50:1750-5. [PubMed: 19824079]

47. Morrill JA, Shrestha M, Grant RW. Barriers to the treatment of hepatitis C. Patient, provider, and system factors. J Gen Intern Med. 2005; 20:754-8. [PubMed: 16050887]

48. Evon D, Simpson K, Esserman D, et al. Barriers to accessing care in patients with chronic hepatitis C: the impact of depression. Aliment Pharmacol Therap. 2010; 32:1163-73. [PubMed: 21039678]

49. McHutchison JG, Bacon BR, Owens GS. Making it happen: managed care considerations in vanquishing hepatitis C. Am J Manag Care. 2007; 13(Suppl 12):S327-36. [PubMed: 18095781]

50. Soto TA, Bell J, Pillen MB. Literature on integrated HIV care: a review. AIDS Care. 2004; 16(Suppl 1):S43-55. [PubMed: 15736821]

51. Fleming CA, Tumilty S, Murray JE, et al. Challenges in the treatment of patients coinfected with HIV and hepatitis C virus: need for team care. Clin Infect Dis. 2005; 40(Suppl 5):S349-54. [PubMed: 15768347]

52. Nguyen HA, Miller AI, Dieperink E, et al. Spectrum of disease in U.S. veteran patients with hepatitis C. Am J Gastroenterol. 2002; 97:1813-20. [PubMed: 12135041]

53. Rifai MA, Indest D, Loftis J, et al. Psychiatric management of the hepatitis C patient. Curr Treat Opt Gastroenterol. 2006; 9:508-19. 
54. Barrera M Jr, Glasgow RE, McKay HG, et al. Do Internet-based support interventions change perceptions of social support?: an experimental trial of approaches for supporting diabetes selfmanagement. Am J Comm Psychol. 2002; 30:637-54. 


\section{WHAT IS CURRENT KNOWLEDGE}

$\checkmark$ A majority of patients with chronic hepatitis $\mathrm{C}$ are deferred from antiviral therapy owing to mental health and substance abuse comorbidities.

$\checkmark$ Retrospective and prospective observational studies suggest that integrated or multidisciplinary treatment models may increase the number of patients who become eligible for, and successfully complete antiviral treatment.

\section{WHAT IS NEW HERE}

$\checkmark$ This is the one of the first studies to test an integrated care model for chronic hepatitis $\mathrm{C}$ patients using a clinical trial design.

$\checkmark$ Novel health service approaches, such as integrated care intervention models, can increase the eligibility for HCV treatment and expand treatment to the underserved population with mental health and substance abuse comorbidities. 


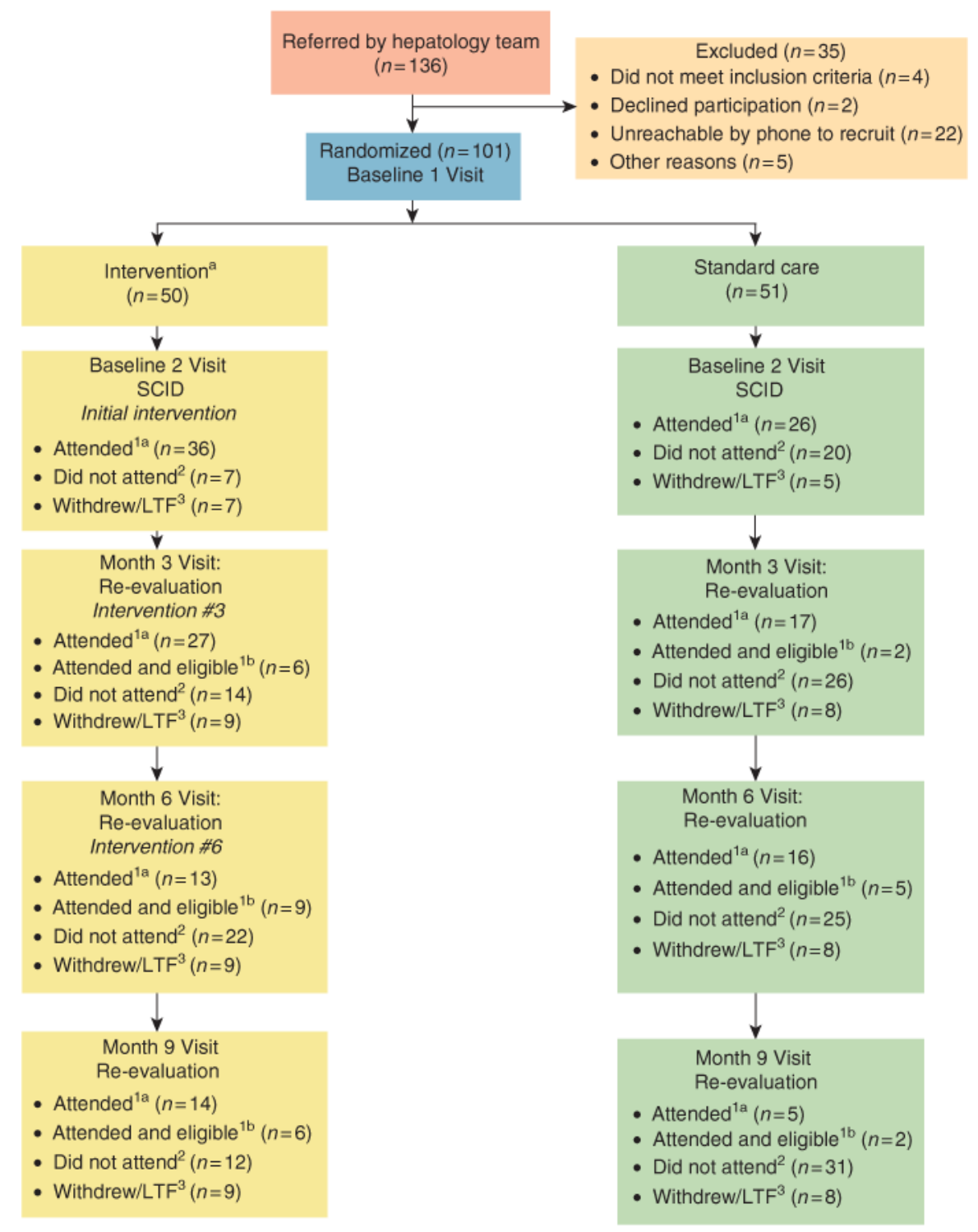

Figure 1.

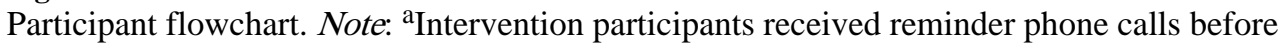
every clinic visit (months 3, 6, and 9) and scheduled phone session (months 1, 2, 4, 5, 7, and 8). ${ }^{1 \mathrm{a}}$ Attended this clinic visit. ${ }^{\mathrm{b}}$ Attended and deemed eligible for treatment at this visit. Numbers not cumulative. ${ }^{2}$ Did not attend this clinic visit, but continued participation in study. Numbers not cumulative. ${ }^{3}$ Withdrawn or lost to follow-up from this point forward. Numbers are cumulative. The number of patients in months 6 and 9 boxes total 50 INT and $51 \mathrm{SC}$ if one adds patients deemed eligible ${ }^{1 \mathrm{~b}}$ at preceding visit(s). INT, integrated care intervention; LTF, lost to follow-up; SC, standard of care; SCID, Structured Clinical Interview for the DSM-IV. 


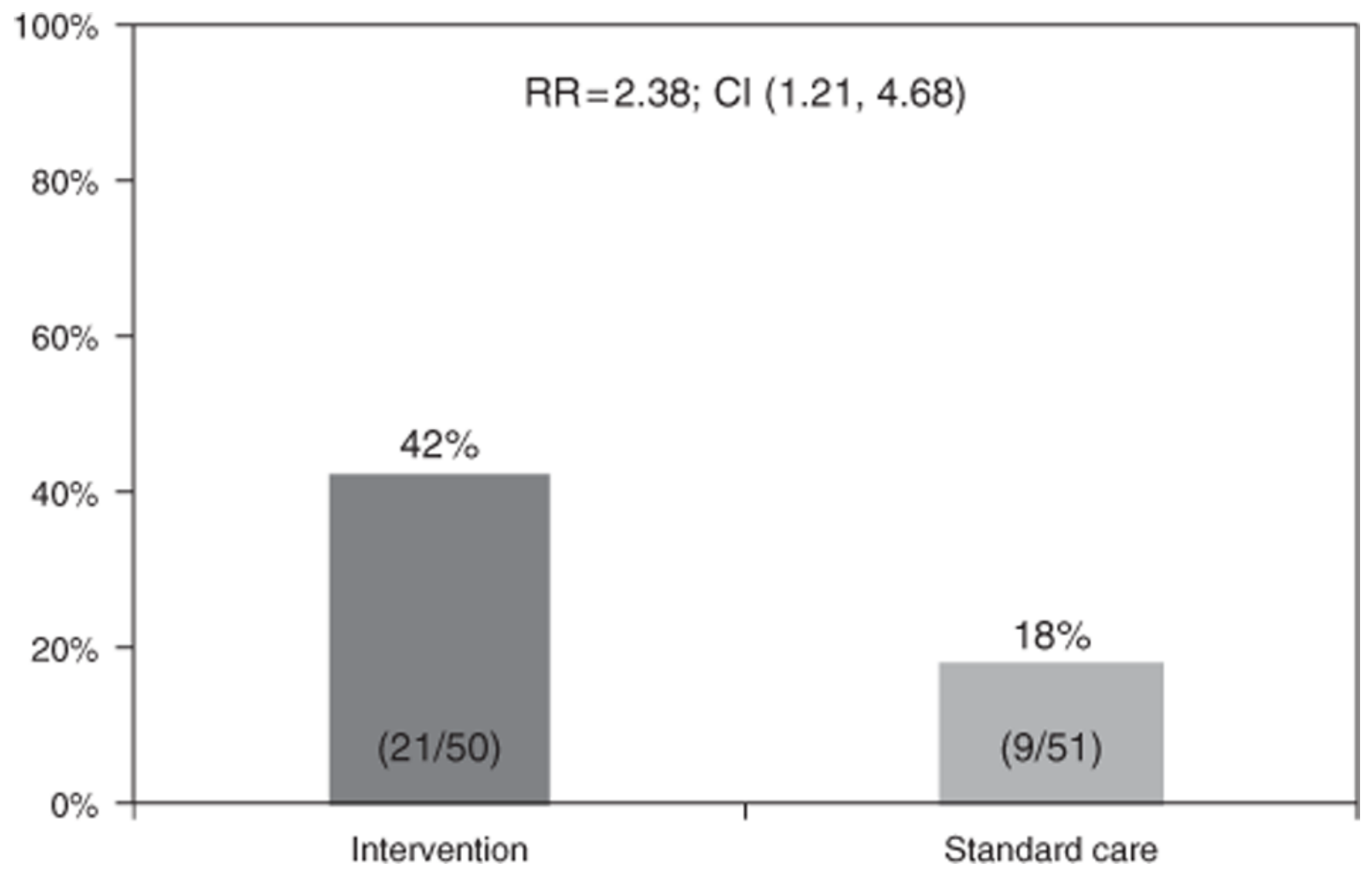

Figure 2.

Intent-to-treat analysis of treatment eligibility rates. CI, confidence interval; RR, relative risk. 
Table 1

Patient demographic and baseline characteristics

\begin{tabular}{|c|c|c|c|}
\hline & $\begin{array}{l}\text { Standard care } \\
\quad(n=51)\end{array}$ & $\begin{array}{c}\text { Intervention } \\
\quad(n=50)\end{array}$ & $P$ value \\
\hline Age mean (s.e.) & $48.3(0.9)$ & $47.7(1.1)$ & 0.69 \\
\hline \multicolumn{4}{|l|}{ Gender, N (\%) } \\
\hline Male & $26(51)$ & $25(50)$ & 1.0 \\
\hline Female & $25(49)$ & $25(50)$ & \\
\hline \multicolumn{4}{|l|}{ Race, N (\%) } \\
\hline Caucasian & $25(50)$ & $36(72)$ & 0.04 \\
\hline Non-Caucasian & $26(50)$ & $14(28)$ & \\
\hline \multicolumn{4}{|l|}{ Marital status, N (\%) } \\
\hline Married/living w partner & $25(49)$ & $23(46)$ & 0.32 \\
\hline Single & $15(29)$ & $10(20)$ & \\
\hline Divorced/separated/widow & $11(22)$ & $17(34)$ & \\
\hline \multicolumn{4}{|l|}{ Education, $\mathrm{N}(\%)$} \\
\hline$<$ HS graduate & $13(26)$ & $19(40)$ & 0.33 \\
\hline HS graduate/GED & $15(30)$ & $12(26)$ & \\
\hline$>$ HS graduate & $22(44)$ & $16(34)$ & \\
\hline \multicolumn{4}{|l|}{ Income, $\mathrm{N}(\%)$} \\
\hline$\leq 20 \mathrm{~K}$ per year & $37(82)$ & $39(83)$ & 1.0 \\
\hline$>20 \mathrm{~K}$ per year & $8(18)$ & $8(17)$ & \\
\hline \multicolumn{4}{|l|}{ Employment status } \\
\hline Full time/part time & $21(43)$ & $21(44)$ & 0.08 \\
\hline Unemployed & $17(35)$ & $7(15)$ & \\
\hline Retired/student/homemaker & $3(6)$ & $6(12)$ & \\
\hline Disabled & $8(16)$ & $14(29)$ & \\
\hline \multicolumn{4}{|l|}{ Insurance status } \\
\hline Private insurance & $17(35)$ & $19(39)$ & 0.93 \\
\hline Medicare/medicaid & $19(39)$ & $18(37)$ & \\
\hline No insurance/hospital charity care & $13(27)$ & $12(24)$ & \\
\hline \multicolumn{4}{|l|}{ Reason for deferral } \\
\hline Alcohol abuse & $18(35)$ & $13(26)$ & 0.77 \\
\hline Substance abuse & $4(8)$ & $5(10)$ & \\
\hline Psychiatric disturbance & $16(31)$ & $19(38)$ & \\
\hline Two or more deferral reasons & $13(25)$ & $13(26)$ & \\
\hline \multicolumn{4}{|l|}{ Genotype } \\
\hline Genotype 1 & $38(74)$ & $39(78)$ & 0.62 \\
\hline Genotype 2 or 3 & $8(16)$ & $11(22)$ & \\
\hline Baseline alcohol mean (s.e.) & $9.2(1.3)$ & $7.5(1.1)$ & 0.33 \\
\hline
\end{tabular}




\begin{tabular}{|lccc|}
\hline & $\begin{array}{c}\text { Standard care } \\
(\boldsymbol{n}=\mathbf{5 1})\end{array}$ & $\begin{array}{c}\text { Intervention } \\
(\boldsymbol{n}=\mathbf{5 0})\end{array}$ & $\boldsymbol{P}$ value \\
\hline Baseline depression mean (s.e.) & $20.0(2.3)$ & $19.9(1.9)$ & 0.96 \\
\hline Baseline anxiety mean (s.e.) & $38.0(2.6)$ & $36.3(1.8)$ & 0.54 \\
\hline Baseline readiness to change mean (s.e.) & $9.79(0.23)$ & $9.03(0.25)$ & 0.03 \\
\hline
\end{tabular}

GED, general educational development; HS, high school graduate.

Note: Missing data were observed for education and employment $(N=97)$; income $(N=92)$; insurance $(N=98)$; genotype $(N=96)$; baseline alcohol, depression, and anxiety $(N=91)$; and baseline readiness to change $(N=88)$. 
Table 2

Multivariable logistic regression models

\begin{tabular}{|llccc|}
\hline Model & Patient characteristic & $\boldsymbol{N}$ & $\mathbf{R R}(\mathbf{9 5 \%}$ CI) & $\boldsymbol{P}$ value \\
\hline 1 & Non-Caucasian & 101 & $0.46(0.20,1.04)$ & 0.06 \\
\hline 2 & Income $\leq 20 \mathrm{~K}$ & 92 & $0.60(0.34,1.05)$ & 0.07 \\
\hline 3 & Genotype 2 or 3 & 96 & $1.69(0.95,3.00)$ & 0.07 \\
\hline 4 & Baseline depression & 91 & $0.98(0.96,1.00)$ & 0.05 \\
\hline 5 & Baseline readiness to change & 88 & $0.90(0.76,1.06)$ & 0.20 \\
\hline
\end{tabular}

CI, confidence interval; RR, relative risk.

Rows present the results of five separate regression models with each containing treatment condition and the patient characteristic noted. The $N$ for each model was reduced in some analyses owing to missing data. 